\title{
Comparing traffic state estimators for mixed human and automated traffic flows
}

\author{
Ren Wang ${ }^{\mathrm{a}}$, Yanning $\mathrm{Li}^{\mathrm{a}}$, Daniel B. Work ${ }^{\mathrm{a}, *}$ \\ ${ }^{a}$ University of Illinois at Urbana-Champaign, Urbana, IL 61801, USA
}

\begin{abstract}
This article addresses the problem of modeling and estimating traffic streams with mixed human operated and automated vehicles. A connection between the generalized Aw Rascle Zhang model and two class traffic flow motivates the choice to model mixed traffic streams with a second order traffic flow model. The traffic state is estimated via a fully nonlinear particle filtering approach, and results are compared to estimates obtained from a particle filter applied to a scalar conservation law. Numerical studies are conducted using the Aimsun micro simulation software to generate the true state to be estimated. The experiments indicate that when the penetration rate of automated vehicles in the traffic stream is variable, the second order model based estimator offers improved accuracy compared to a scalar modeling abstraction. When the variability of the penetration rate decreases, the first order model based filters offer similar performance.
\end{abstract}

Keywords: Traffic state estimation, automated vehicles, second order traffic flow model

\section{Introduction}

The recent viability of vehicle automation and communication systems (VACS) has motivated a growing interest to understand how modern transportation systems will evolve when the technologies are available at scale. Although the dream was first illustrated by General Motors as early as 1939 at the Highways \& Horizons exhibit at the New York World's Fair, widespread commercial research and development of automated vehicles was brought to fruition following the DARPA Grand Challenges in the 2000's. An overview of other key historical developments in vehicle automation and communication systems (e.g., automated highways [1,2], automated vehicle control [3]) leading to the state of practice today can be found in the reviews $[4-7]$.

With the emergence of mixed traffic flows now eminent, the problems of modelling, estimating, and managing mixed traffic streams is now a pressing concern. Unlike human piloted vehicles, automated vehicles (AVs) have the capability to significantly reduce the

\footnotetext{
*Corresponding author

Email address: dbwork@illinois.edu (Daniel B. Work)
} 
headway between vehicles, potentially adding capacity without increasing the physical infrastructure. Because the AVs may have significantly different operating characteristics compared to the human operated vehicles, an open question is how to model and estimate traffic conditions when the flow is composed of a mix of VACS and non-VACS vehicles. Studies addressing various aspects of the modeling problem include [8-14]. The articles [12, 13] propose a gas-kinetic macroscopic model to describe the operations of cooperative adaptive cruise control (CACC) traffic flow and adaptive cruise control traffic flow respectively. The work [15] contains a detailed review of the various approaches to model VACS in the traffic stream. Several studies also investigate the stability of traffic under various automation and connectivity considerations $[6,8,16,17]$.

In contrast, this article concerns the problem of combining real-time data streams with a (macroscopic) model of mixed AV and human piloted traffic to generate traffic state estimates. Classically, such problems for non-VACS traffic are posed as a sequential state estimation problem based on the state space form of the model and measurement system:

$$
\begin{aligned}
x^{n+1} & =f\left(x^{n}, \omega^{n}\right) \\
z^{n} & =h\left(x^{n}, \nu^{n}\right),
\end{aligned}
$$

where $x^{n}$ denotes the traffic state at discrete time step $n$ such as a vector of the density of traffic along the roadway, and $f$ is a traffic flow model that evolves the traffic state from one time step to the next. The model noise $\omega^{n}$ is a random variable that represents the one time step prediction error of the model. The function $h$ is known as the observation equation and defines how the vector of measurements $z^{n}$ received at time $n$ is related to the traffic state variable $x^{n}$, and $\nu^{n}$ is a random variable describing the measurement error.

The problem of estimating the traffic state vector $x^{n}$ using measurements $Z^{n}=\left\{z^{1}, \cdots, z^{n}\right\}$ and the system (1) was introduced with the early works of Gazis and Knapp [18] and Setzo and Gazis [19] in the 1970's through Kalman filtering and its extensions applied to data collected on the Lincoln Tunnel in New York City. Beginning in the early 1980's, a modified version of Payne's macroscopic model has been used for a variety of Kalman-based estimators [20-23]. Nonlinear variants of Kalman filtering [24-29] and particle filtering [30-35] have also been applied to modifications of the Lighthill Whitham Richards (LWR) partial differential equation (PDE) and its discretization [36-39].

Compared to human operated traffic without connectivity, filtering based approaches for traffic containing connected or automated vehicles is still relatively unexplored. The most closely related approaches are works of Bekiaris-Liberis et al. [40, 41] and Roncoli et al. [42], which design a traffic state estimator under two key assumptions on mixed VACS and human operated vehicle flows. First, a scalar traffic flow model is used in the traffic evolution equation in which the velocity field along the roadway is treated as a known timevarying parameter, and is assumed to be provided by the connected vehicles in the traffic stream.

A second important assumption is that the velocity measurements from the automated vehicles are assumed to be representative of the velocity of all vehicle types. This assumption is motivated by the fact that in free flow, both vehicle types will have the same average speed (e.g., as established by the speed limit), and in congestion, the VACS are obstructed by other 
(human operated) vehicles and consequently adapt the same speed due to the difficulty of overtaking. This assumption is a critical one, and will be exploited in the present work to establish the connection of mixed automated and human operated traffic flow with a generalization of the Aw Rascle Zhang (ARZ) [43, 44] model.

Under the two assumptions above, the state-space model and its observability is analyzed in [40, 41], and numerical experiments are performed using the macroscopic METANET model describing average density and speeds as the true state. Experiments validated on field data under various penetration rates are explored using the NGSIM dataset in [42]. Such a validation is appropriate if the mixed traffic stream is composed of VACS which drive at similar speeds and spacings as human operated vehicles, as is potentially the case for many connected vehicle systems. Other estimation approaches [45, 46] exploit additional sensor data available from some VACS to improve the traffic state estimates.

In the same general theme of [40-42], in this work we compare two different models to predict the traffic state within an estimator when traffic is composed of human piloted and automated vehicles. One approach uses the classical LWR model, which specifies that vehicles are conserved and the speed of traffic is related to the total density of human and automated vehicles in the traffic stream. The second model uses a variant of the second order ARZ traffic flow model, known as the collapsed generalized ARZ model [47]. Note these models are more accurately referred to as $2 \times 2$ systems of conservation laws, but we adopt the common name "second order" in this article. Recently, a connection between second order models and two-class traffic flow models was established [48], thereby motivating the use of the ARZ model for application for two-class traffic. Recognizing automated vehicles and human operated vehicles as two separate classes of traffic, the ARZ modeling framework is a natural modeling approach to predict the evolution of the traffic state. Other multi-class traffic flow models, such as [49-51] may be appropriate if overtaking is determined to be a critical feature of the mixed human and AV flow.

The main question addressed in this article is to what extent the additional modeling detail provided by two-class (equivalently ARZ) models can enhance the traffic estimates of mixed traffic flows. The estimation comparison is conducted in a micro simulation environment, where a subset of the vehicles are identified as automated, and consequently their properties are distinct from the vehicles simulated under typical human operated characteristics. Note in the present work we focus only on automated vehicles, and do not explore connectivity between vehicles in the traffic stream. Connectivity can create non-local effects in the traffic stream (e.g., advanced warning of slow downs) that are treated, for example in the works $[52,53]$. In this work, automated vehicles are assumed to use only local information to operate the vehicle, thereby eliminating non-local effects.

The contributions of this article are summarized as follows:

- The applicability of the second order traffic flow model for modeling mixed human and automated traffic is established under the assumption of limited overtaking between traffic flows.

- The estimation performance of the second order traffic model and the first order traffic model are compared by implementing a particle filter on mixed human and automated 
traffic generated in a micro simulation environment. It is shown that when the variability of the penetration rate increases, the second order model based estimator offers superior estimation performance. At lower rates, well calibrated first order model abstractions offer similar estimation performance.

The reminder of the article is organized as follows. In Section 2, both the first and the second order traffic flow models are reviewed. In Section 3, we introduce the particle filter, which is used as the nonlinear traffic state estimation algorithm in this work. Numerical experiments are performed in Section 4, where the estimation performance of the first and second order traffic models are compared. Conclusions are summarized in Section 5.

\section{Macroscopic traffic models}

In this section, two models are proposed for use within a model based estimator for mixed automated and human piloted traffic. The first and coarsest model is the seminal LWR model [36, 37], which assumes that the total traffic density evolves according to a conservation law and a constitutive model of the traffic speed as a function of the traffic density. The effects of automated vehicles in the traffic stream are assumed to be entirely captured by the shape and parameters of the fundamental diagram. A more refined second order traffic flow model known as the generalized Aw Rascle Zhang (GARZ) [54] is also applied to model mixed traffic. The GARZ allows the fundamental diagram to be further adjusted based on the fraction of AVs in the traffic stream, as will be explained in detail.

\subsection{First order traffic model}

We briefly review the LWR model $[36,37]$, which is a conservation law governing the evolution of the traffic density $\rho(\chi, t) \in\left[0, \rho_{\max }\right]$ at location $\chi$ and at time $t$ on a roadway:

$$
\begin{aligned}
& \frac{\partial \rho(\chi, t)}{\partial t}+\frac{\partial(\rho(\chi, t) v(\rho(\chi, t)))}{\partial \chi}=0, \\
& (\chi, t) \in(0, L) \times(0, T) .
\end{aligned}
$$

The initial and (weak) boundary conditions are specified as follows:

$$
\rho(\chi, 0)=\rho_{0}(\chi), \rho(0, t)=\rho_{l}(t), \rho(L, t)=\rho_{r}(t),
$$

where $\rho_{0}, \rho_{l}$, and $\rho_{r}$ are the initial, left, and right traffic density boundary conditions.

The LWR model requires a closure assumption that the velocity of traffic $v$ is a function of the density of traffic only. Under this assumption, an empirical relationship between the velocity and density is constructed and used to complete the model. While many velocity functions are available, in this work the following quadratic-linear function proposed by 
Smulders [55] is adopted:

$$
v(\rho)= \begin{cases}v_{\max }\left(1-\frac{\rho}{\beta}\right) & \text { if } \rho \leq \rho_{c} \\ \frac{v_{\max } \rho_{c}\left(\rho_{m}-\rho\right)\left(\beta-\rho_{c}\right)}{\rho \beta\left(\rho_{m}-\rho_{c}\right)} & \text { otherwise. }\end{cases}
$$

In (4), the variable $v_{\max }$ denotes the maximum speed that vehicles can travel on the road. The parameter $\beta$ determines the curvature of the velocity function for the free flow regime. In particular, it determines how the average vehicle speed will change when the traffic density increases from zero to the critical density $\rho_{c}$. The critical density is the traffic density when the highway has the maximum traffic flow. The variable $\rho_{m}$ denotes the jam density, which corresponds to the traffic density when the road is completely congested. The critical density and jam density influence the shape of the velocity function for the congested regime.

When applied to model mixed human and automated traffic, the LWR model tracks only the total density of vehicles on the roadway, independent of the vehicle type (e.g., human or VACS). It assumes that the speed of traffic can be determined by only examining the local density of all traffic.

\subsection{Second order traffic model}

\subsubsection{Generic framework of a second order traffic model}

A generalized ARZ model [54] that fits into the framework of the generic second order model (GSOM) [56] is proposed to describe the evolution of mixed human and automated traffic on the highway. In conservative form, the model reads:

$$
\begin{aligned}
& \frac{\partial \rho}{\partial t}+\frac{\partial(\rho v(\rho, w))}{\partial \chi}=0 \\
& \frac{\partial y}{\partial t}+\frac{\partial(y v(\rho, w))}{\partial \chi}=0 \\
& (\chi, t) \in(0, L) \times(0, T)
\end{aligned}
$$

where $w$ is known as a property. The second equation indicates that the total property $y=\rho w$ is conserved, along with vehicle conservation as specified in the first equation. The function $v(\cdot, \cdot)$ denotes the velocity function for the second order traffic model, which is parametrized both by the density (as in the first order model), and by the property.

The weak boundary conditions of (5) are given by:

$$
\begin{aligned}
& \rho(\chi, 0)=\rho_{0}(\chi), \rho(0, t)=\rho_{l}(t), \rho(L, t)=\rho_{r}(t), \\
& y(\chi, 0)=y_{0}(\chi), y(0, t)=y_{l}(t), y(L, t)=y_{r}(t),
\end{aligned}
$$

where the variables $y_{0}, y_{l}$, and $y_{r}$ are the initial, left, and right boundary conditions for the total property $y$. 
In (7), the second equation indicates that $w$ is advected with vehicles at the speed of $v$, and thus the property is conserved along vehicle trajectories. Depending on the application, further interpretations of the property variable $w$ have been proposed for human piloted traffic, such as "aggressivity" [54], "desired spacing" [57], or equilibrium "perturbations" [58].

In this work, we propose to define the property $w \in[0,1]$ as the fraction of automated vehicles in the traffic stream (equivalently the penetration rate of $\mathrm{AVs}$ ). In principle, $\mathrm{AVs}$ may operate with a much smaller spacing compared to regular traffic, due to the fact that the perception-interpretation-reaction time of an AV may be significantly reduced compared to humans. As a result, the velocity function (and therefore the fundamental diagram) depends not only on the density of traffic, but also the composition of the flow, as indicated by $w$. The larger the fraction of AVs, the smaller the spacing between vehicles at a given speed, and consequently a larger flow can be maintained.

By defining $w$ as the fraction of $\mathrm{AVs}$, conservation of $\rho_{a}=y=w \rho$ as specified in the second equation of (5) denotes conservation of the automated vehicles. Let $\rho_{h}$ and $\rho_{a}$ denote the density of human piloted and automated vehicles respectively, where $\rho=\rho_{h}+\rho_{a}$ and $w=\rho_{a} / \rho$. Under these definitions the model (5) becomes:

$$
\begin{aligned}
& \frac{\partial \rho}{\partial t}+\frac{\partial\left(\rho v\left(\rho, \rho_{a} / \rho\right)\right)}{\partial \chi}=0, \\
& \frac{\partial \rho_{a}}{\partial t}+\frac{\partial\left(\rho_{a} v\left(\rho, \rho_{a} / \rho\right)\right)}{\partial \chi}=0, \\
& (\chi, t) \in(0, L) \times(0, T),
\end{aligned}
$$

which expresses conservation of all vehicles and conservation of the automated vehicles.

By subtracting the second equation (8) from the first, we find the human piloted vehicles 
$\rho_{h}$ must also be conserved. As a result, (8) is equivalent to:

$$
\begin{aligned}
& \frac{\partial \rho_{h}}{\partial t}+\frac{\partial\left(\rho_{h} \tilde{v}\left(\rho_{h}, \rho_{a}\right)\right)}{\partial \chi}=0 \\
& \frac{\partial \rho_{a}}{\partial t}+\frac{\partial\left(\rho_{a} \tilde{v}\left(\rho_{h}, \rho_{a}\right)\right)}{\partial \chi}=0 \\
& (\chi, t) \in(0, L) \times(0, T) .
\end{aligned}
$$

where the velocity function $\tilde{v}$ is defined as follows:

$$
\tilde{v}\left(\rho_{h}, \rho_{a}\right)=v\left(\rho_{h}+\rho_{a}, \frac{\rho_{a}}{\rho_{h}+\rho_{a}}\right) .
$$

The model (9) establishes equivalence for the GARZ model (5) [56] as a two-class conservation law model for human driven and automated traffic. From (9), it is noted that both vehicle classes choose the same speed when the composition of traffic (i.e., the density of automated and human driven vehicles) is fixed. It is therefore also equivalent to the generic two class model proposed by Zhang and Jin [59].

The model above makes explicit the overtaking assumptions stated in [40-42]. In light traffic, both vehicle classes travel at the same speed given a fixed density of each vehicle class because the velocity of traffic is effectively governed by the speed limit. In congested traffic, the speed of each vehicle class is fixed based on the average speed of the traffic surrounding the vehicles. Large rates of overtaking by one class is not possible in congestion without dedicated lanes for a preferred vehicle class, which is assumed to be unavailable.

Another motivation for (10) to depend on both class densities is as follows. Given a fixed speed of traffic, the main difference is the spacing (equivalently density) of the two vehicle types. When the total density is composed primarily of human piloted vehicles, the average spacing will be larger (i.e., the density is smaller) than when the total density is composed of primarily automated traffic.

Interestingly, if the fraction of $\mathrm{AVs}$ in the traffic flow is constant in space and time, the model reduces to the LWR PDE [36, 37], where the velocity depends only on total density (i.e., the fraction $w$ becomes a parameter). Thus, the LWR PDE can be viewed as a special form of the GARZ, with a uniform fraction of AVs $w(\chi, t)=\bar{w}$ (i.e., $v(\rho)=v(\rho, \bar{w})$ ). This further motivates the use of the LWR model (2) as a forward model in an estimation framework when the penetration rate of $\mathrm{AVs}$ is approximately constant. This intuition is verified in the numerical experiments in Section 4.

The velocity function $v$ (or $\tilde{v}$ ) needs to be specified to complete the mixed human and automated traffic model. Different types of velocity functions have been used based on the assumptions on the property quantity $w$. The models proposed by Aw and Rascle [43] and Zhang [44] and the GARZ [54] allow the driver property to influence the velocity function both in the freeflow and congested regimes. However, in this work, a quadratic-linear velocity function developed based on the collapsed GARZ (CGARZ) [60] is deployed, in 
which the composition of the flow does not influence the free flow speed:

$$
v(\rho, w)= \begin{cases}v_{\max }\left(1-\frac{\rho}{\beta}\right) & \text { if } \rho \leq \rho_{c}(w) \\ \frac{v_{\max } \rho_{c}(w)\left(\rho_{m}-\rho\right)\left(\beta-\rho_{c}(w)\right)}{\rho \beta\left(\rho_{m}-\rho_{c}(w)\right)} & \text { otherwise. }\end{cases}
$$

The velocity function (11) is a second order extension of the first order velocity function (4), and again the variables $v_{\max }$ and $\beta$ determine the shape of the velocity function for the free flow regime. The fact that the velocity function in the free flow regime does not depend on the fraction of AVs defines the model as a collapsed GARZ model whose well posedness is established in [60]. All vehicles are assumed to drive near the free flow speed regardless of their property $w$. The variables $\rho_{c}$ and $\rho_{m}$ are the critical density and jam density for the second order traffic model, and determine the shape of the second order velocity function in the congested regime. Note the critical density is different depending on the fraction of AVs, so the parameter $\rho_{c}$ depends on the fraction $w$. Assuming the human piloted and autonomous vehicles have the same average vehicle length and average minimum gap (i.e., minimum front bumper to lead vehicle rear bumper distance), then the jam density of traffic does not depend on the composition of AVs in the traffic stream.

For integration in an online estimator, the continuum flow models are discretized according to a Godunov scheme as detailed in the Appendix.

\section{Traffic estimation}

In order to compare the performance of the first and second order traffic models for traffic estimation, a standard particle filter $[61,62]$ is deployed with both models. The particle filter is a fully nonlinear Bayesian estimator and is subject only to a Monte Carlo approximation error instead of Gaussianity or unimodal approximations exploited by minimal variance Kalman-based estimators.

In this work, the model parameters in the fundamental diagram are calibrated offline, and consequently only the state estimation problem is considered. An alternative approach is to jointly estimate the parameters with the state variables online, for example following an approach similar to [22]. However, due to the structure of the ARZ model, some fundamental diagram parameters may not be observable depending on the observation equation, which may result in parameter divergence. Consequently in this work we study the simplified setting of offline model calibration and online state estimation.

\subsection{Particle filter}

The sequential state estimation problem for the state space model (1) is formulated using the Bayesian approach [63]. This approach estimates the posterior probability density function $p\left(x^{n} \mid Z^{n}\right)$, where $x^{n}$ is the system state and $Z^{n}$ are the measurements from time step one to time step $n$. In the first order model, the system state is the vector of the total density in each cell, $x^{n}=\left[\rho_{1}^{n}, \cdots, \rho_{i_{\max }}^{n}\right]^{T}$. For the second order model the state is the total density 
and the density of automated vehicles, $x=\left[\rho_{1}, \cdots, \rho_{i_{\max }}^{n},\left(\rho_{a}\right)_{1}^{n}, \cdots,\left(\rho_{a}\right)_{i_{\max }}^{n}\right]^{T}$. In either system, the state vector $x^{n}$ is recursively updated according to:

$$
\begin{aligned}
& p\left(x^{n} \mid Z^{n-1}\right)=\int p\left(x^{n} \mid x^{n-1}\right) p\left(x^{n-1} \mid Z^{n-1}\right) d x^{n-1}, \\
& p\left(x^{n} \mid Z^{n}\right)=\frac{p\left(z^{n} \mid x^{n}\right) p\left(x^{n} \mid Z^{n-1}\right)}{p\left(z^{n} \mid Z^{n-1}\right)} .
\end{aligned}
$$

The first equation of (12) describes the state propagation step from time $n-1$ to time $n$, where $p\left(x^{n-1} \mid Z^{n-1}\right)$ is the posterior distribution at time $n-1$ and $p\left(x^{n} \mid Z^{n-1}\right)$ is the prior distribution at time $n$. In this problem, the system state evolution $p\left(x^{n} \mid x^{n-1}\right)$ is determined by the traffic flow model $f$. In the case of the first order model based estimator, it is the CTM (18), while for the second order model based estimator, it is the 2CTM (22). The second equation is the measurement processing step. When the measurements at time $n$, $z^{n}$, become available, they are used to determine the posterior distribution of the system state $x$ at time $n$. Here, the term $p\left(z^{n} \mid Z^{n-1}\right)$ is a normalizing constant and $p\left(z^{n} \mid x^{n}\right)$ is the likelihood function, which is determined by comparing how well the predicted system state matches with the measurements. Construction of the likelihood function exploits the observation equation in (1). Typically in traffic monitoring applications, the observation equation is straightforward to construct and depends on the type of measurements to be collected (e.g., velocity, density, flow), it's relationship to the state variable (e.g., density), and the location of the measurement.

The particle filter approximates the probability densities in (12) with samples, and uses a Monte Carlo integration to compute the prior distribution at time $n$ given the posterior at time $n-1$. A pseudo-code and summary of a standard formulation of the particle filtering algorithm is provided in the Appendix.

\section{Numerical simulation}

To assess the accuracy of the models when used within an estimator, we conduct a series of numerical experiments based on micro simulated traffic containing a mix of human piloted and automated vehicles. The overview of the numerical simulations is as follows. First, we describe the roadway setup and vehicle parameter settings used to generate mixed traffic flow. Then, the process of calibrating the first order and second order traffic flow models for mixed traffic is presented. Next, a traffic scenario is carefully constructed to contain a mix of freeflow and congested traffic conditions, and the performance of the estimators is quantified. Note that by construction, first and second order models are expected to perform similarly in free flowing traffic far from any congestion due to the collapsed nature of the fundamental diagram (i.e., the light traffic velocity depends only on the total density and is independent of $w$ ).

The numerical simulation is conducted in Aimsun, which is a microscopic traffic simulation software. A three-mile segment of a two-lane freeway is modeled in Aimsun for the 
experiment. The speed limit of the roadway is assumed to be 70 miles per hour. The simulation time step used within Aimsun to resolve the vehicle trajectories is set at 0.2 seconds. Aimsun has previously been used to simulate adaptive cruise control vehicles (e.g., laterally automated vehicles) in [64]. In this work, we adopt a simpler approach, where the automated vehicles are assumed to drive sufficiently like humans, only with enhanced capabilities (e.g., reduced perception reaction time, desired time headway, etc.).

Two classes of vehicles are simulated, namely, human piloted vehicles and AVs. The AV class is created in Aimsun based on the car class with the following parameters changed to mimic a plausible behavior of AVs. The first parameter is the reaction time, which determines the reaction time of a vehicle to the speed change of its preceding vehicle. The reaction time directly affects the headway in accelerating traffic (e.g., clearance of a queue). In this work, the reaction time of $\mathrm{AVs}$ is modified to be $0.2 \mathrm{~s}$ while the reaction time of humans is $0.8 s$, which are slightly larger values used in [64]. The second parameter is the minimum headway, which should not to be confused with the headway. The minimum headway is used in the deceleration component of a following vehicle to guarantee a safe time gap to its preceding vehicle. The minimum headway of AVs is specified as $0.8 s$ while the minimum headway of human piloted vehicles is specified as $2 \mathrm{~s}$. These modified microscopic parameters indeed result in reasonable change in macroscopic characteristics (e.g., an increase in capacity is observed when penetration rate of AVs increase), which is consistent with [64].

Finally, the human-related stochasticity of parameters are removed from the AVs by setting the standard deviation of the appropriate parameters to 0. Such parameters include the speed acceptance and the minimum stopping distance. The nominal values of such parameters are set at the mean values of the car class and are described in more detail in the supporting source code and Readme (https://github.com/Lab-Work/AIMSUN_with_AVs). Higher fidelity micro simulation models of automated traffic [16, 64-66] may be added to modify the capabilities of the automated vehicle flow in the simulation.

\subsection{Fundamental diagram calibration}

In this section, the calibration procedure to determine the fundamental diagram parameters for both the first order model (2) and the second order model (7) is described. Given the velocity function (11), the fundamental diagram for the second order model is written as:

$$
q(\rho, w)= \begin{cases}v_{\max }\left(\rho-\frac{\rho^{2}}{\beta}\right) & \text { if } \rho \leq \rho_{c}(w), \\ \gamma(w)\left(\rho-\rho_{m}\right) & \text { otherwise. }\end{cases}
$$

In formulation (13), $q(\rho, w)$ is assumed to be a quadratic function in freeflow and a linear function in congestion where $\gamma(w)=\left(-v_{\max } \rho_{c}(w)\left(\beta-\rho_{c}(w)\right)\right) /\left(\beta\left(\rho_{m}-\rho_{c}(w)\right)\right)$ denotes the maximum backward propagation speed of a shock wave given a traffic stream with a fraction of automated vehicles specified by $w$. The first order fundamental diagram is constructed similarly, with the simplification that the parameters do not depend on $w$.

To determine the parameters of the fundamental diagram, traffic data is collected from detectors in Aimsun with a detection interval $20 \mathrm{~s}$, at different penetration rates of AVs in 

of free flowing and congested traffic conditions. This data mimics a historical sensor dataset that may be used for calibration, which would be commonly available in a field deployment.

In the free flow regime, the parameter $\beta$ is first selected as $600 \mathrm{veh} / \mathrm{mile}$ to approximate the quadratic function to a linear function. Then the parameter $v_{\max }$ is obtained by a least squares regression on the free flow data collected for all penetration rates of AVs. For the congested flow regime, the jam density is analytically computed by assuming mean minimum stopping distance and average vehicle length as $\rho_{m}=644$ veh $/$ mile, regardless of the penetration rate of AVs. Then the maximum backward shock wave propagation speed $\gamma(w)$ is obtained by a linear regression on the congested flow data collected for each penetration rate of $\operatorname{AVs} w$. The critical density $\rho_{c}(w)$ and the maximum flow $q_{\max }(w)$ are obtained by computing the intersection between the collapsed quadratic function in the free flow regime and the linear function in the congested flow regime for each $w$.

The first order fundamental diagram is assumed to share the same parameters $v_{\max }$ and $\beta$ with the second order fundamental diagram (13) in the free flow regime. The jam density is analytically computed as $\rho_{m}=644 \mathrm{veh} / \mathrm{mile}$ as in the second order model. The parameter $\gamma$ in the first order fundamental diagram does not depend on $w$, but is recalibrated for simulation scenario. For example, if the penetration rate of $\mathrm{AVs}$ in the inflow in a simulation scenario is expected to vary in $0 \% \sim 30 \%$, then the data points with $w \in[0.0,0.3]$ previously collected for calibration are used to determine $\gamma$ by a least squares regression. In other words, we assume the historical data used to fit the fundamental diagram parameters in the first order model is representative (with respect to the variability of the AV penetration rate) of the traffic conditions for which the estimator is applied. In other scenarios with different ranges of expected penetration rate of $\mathrm{AVs}$, the parameter $\gamma$ is updated with the corresponding calibration dataset. The calibration of $\gamma$ determines the critical density and the capacity of the first order fundamental diagram.

\subsection{Simulation description}

Numerical simulations are conducted to evaluate how the estimators perform for traffic with different variability of the penetration rate of AVs. In total, five simulation scenarios are evaluated, with the penetration rate of $\mathrm{AVs}$ in the upstream inflow sampled from uniform distributions $\mathcal{U}(0,0), \mathcal{U}(0,0.25), \mathcal{U}(0,0.5), \mathcal{U}(0,0.75), \mathcal{U}(0,1)$ where the variability of the penetration rate (i.e., the range of the uniform distribution) are respectively $0 \%, 25 \%, 50 \%, 75 \%, 100 \%$. Each replication simulates 60 minutes traffic on the three-mile freeway.

For each simulation, the upstream traffic flow is set by a piecewise constant function with 12 five-minute intervals. The fraction of $\mathrm{AVs}$ in each interval is randomly sampled from a uniform distribution specified by the scenario. Then the upstream flow in each interval is set close to the maximum capacity, ranging from 3,600 to $6,600 \mathrm{veh} / \mathrm{hr}$ depending on the penetration rate of AVs. The inflow is selected as a function of the AV penetration rate so that congestion is always obtained in each simulation. In each simulation, the roadway is initially empty. On the downstream boundary of the domain, congestion is induced 


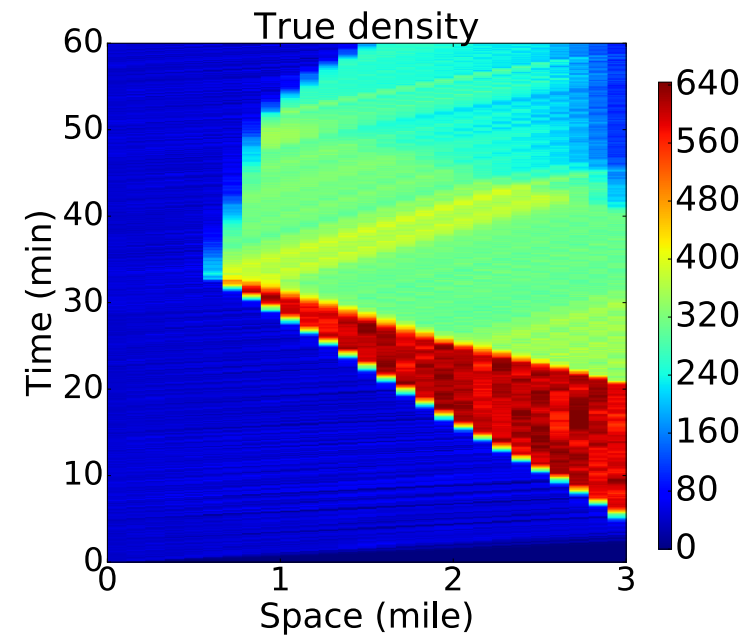

(a)

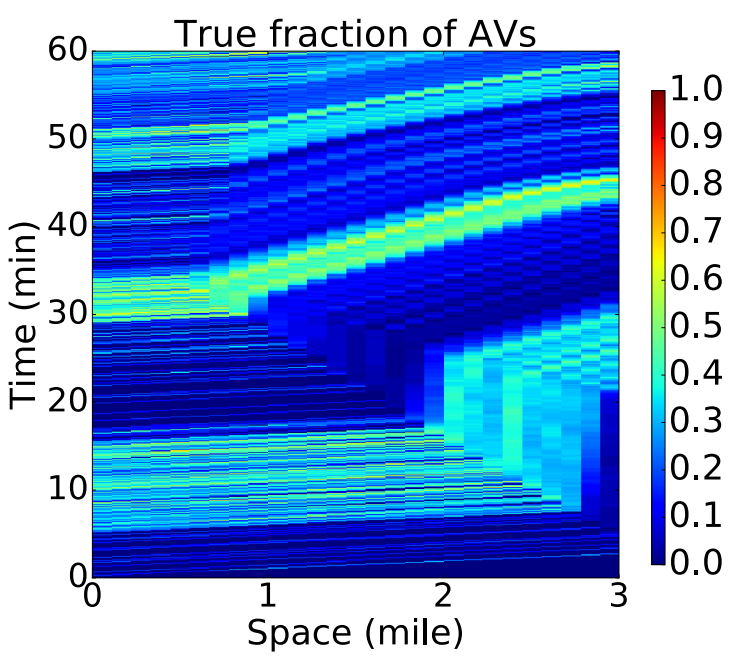

(b)

Figure 1: An example of the simulated true total traffic density $\rho$ (veh/mile) with the penetration rate of AVs sampled from $\mathcal{U}(0,0.5)$.

through a downstream bottleneck which reduces the outflow of the estimation domain. More precisely, a time varying speed limit is set downstream such that the outflow for the first 20 minutes is limited to approximately $300 \mathrm{veh} / \mathrm{hr}$, which triggers a congestion wave in the estimation domain. Then during 20 to 40 minutes, the outflow increases to approximately $3000 \mathrm{veh} / \mathrm{hr}$ which represents a clearance phase of the congestion. Finally, after 40 minutes, the downstream bottleneck completely removed. The resulting heavy traffic grows until the congestion clearance phase, and an expansion wave begins to dissipate the congestion from the front of the bottleneck. Eventually the congestion dissipates and the traffic flow returns to a freeflow condition. An example of the aggregated traffic conditions when the fraction of $\mathrm{AVs}$ in the inflow is sampled from $\mathcal{U}(0,0.5)$ is shown in Figure 1. Figure 1 plots the true total traffic density $\rho$ and penetration rate $w$, and exhibits the growth and clearing of the congestion that results in a nontrivial traffic state for the estimator to recover.

The above setup is designed to include the relevant effects of the bottleneck inside the estimation domain. Specifically, the true traffic state includes the following: $i$ ) the transition of traffic from free flow to congestion (i.e., the back of the queue); ii) the traffic state in the queue caused by the bottleneck; and iii) the transition from congestion to freeflow when the bottleneck is removed (i.e., the dissipation wave from the front of the queue). The state far downstream from the bottleneck will be in free flow assuming no further restrictions in capacity, which is not particularly interesting from an estimation standpoint. Consequently, the current simulation provides a good range of traffic conditions that reflect the challenging transitions for the estimation problem.

The true total density $(\rho)$, and the true fraction of $\operatorname{AVs}(w)$ of the simulated traffic are computed from the Aimsun trajectory data. The aggregated quantities are defined on 
time-space grid with $\Delta \chi=179 m$ and $\Delta T=5 s$ based on Edie's definitions [67] as follows:

$$
\begin{gathered}
\rho=\frac{\sum_{j} \tau_{j}}{\Delta \chi \Delta T}, \\
w=\frac{\sum_{j} \tau_{j} \mathcal{I}(j)}{\sum \tau_{j}},
\end{gathered}
$$

where $\tau_{j}$ is the time spent by the vehicle $j$ in each time space cell, and the sums are over the vehicle trajectories that appear in the cell. The indicator function $\mathcal{I}$ in (15) is defined as:

$$
\mathcal{I}(j)= \begin{cases}1 & \text { if } j^{\text {th }} \text { vehicle is } \mathrm{AV} \\ 0 & \text { otherwise. }\end{cases}
$$

From Aimsun, a limited measurement data is extracted and made available to the estimator to emphasize the differences between the first and second order models used within the estimator. Specifically, the measurements are collected by four traffic detectors deployed on the freeway at a one mile spacing. The detectors measure the traffic flow and speed which are then processed to obtain the density measurement. The vector of density measurements result in a linear observation equation (1). At the boundaries, the density and property measurements are used as inputs to the state space model.

To determine the performance of the estimator on a given true state realization, the standard mean absolute error is used as the metric and computed for each simulation scenario. Let $\rho^{n}$ denote the vector of true total density state at time $n$ as computed by (14), and let $\hat{\rho}^{n}$ denote the estimate of the true density state according to the particle filter applied to the first or second order traffic model. The mean absolute error is computed as:

$$
e=\frac{1}{\sum_{n=0}^{n_{\max }} \sum_{i=0}^{i_{\max }}} \sum_{n=0}^{n_{\max }} \sum_{i=0}^{i_{\max }}\left|\rho_{i}^{n}-\hat{\rho}_{i}^{n}\right| .
$$

All simulations are performed using Aimsun 8.0.9 on a desktop with a quadcore Intel i7-4770 3.4 GHz processor. Ten replications of microscopic traffic conditions are simulated for each variability scenario, where the fraction of $\mathrm{AVs}$ in the inflow (divided into 12 intervals) of each micro simulation replication is drawn from the corresponding distribution (e.g., $\mathcal{U}(0,0.25))$. Each microsimulation takes approximately 15 minutes to run the simulation and extract the true state, resulting in a total of 12 hours of micro simulation and data extraction time. For each micro simulation, two particle filtering traffic estimators (one scalar model, and one second order model) are run ten times each. Because the particle filter is a stochastic filter, multiple runs are necessary to get an assessment of the average performance of the PF. The total number of estimation runs is (five penetration rate variabilities) $\times(10$ simulations per penetration rate variability) $\times$ (two estimators per penetration rate $) \times$ (10 particle filter runs per estimator $)=1,000$ particle filter runs. The particle filtering algorithm runs faster than real time on this network, and the 1,000 runs finish in about eight hours on a machine with an eight-core Intel i7-6900k 3.7 Ghz processor. The total 


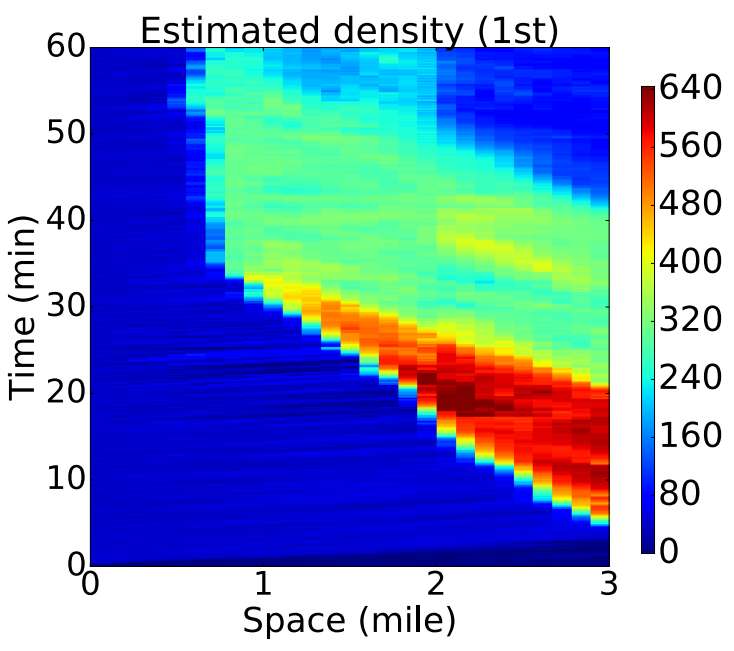

(a)

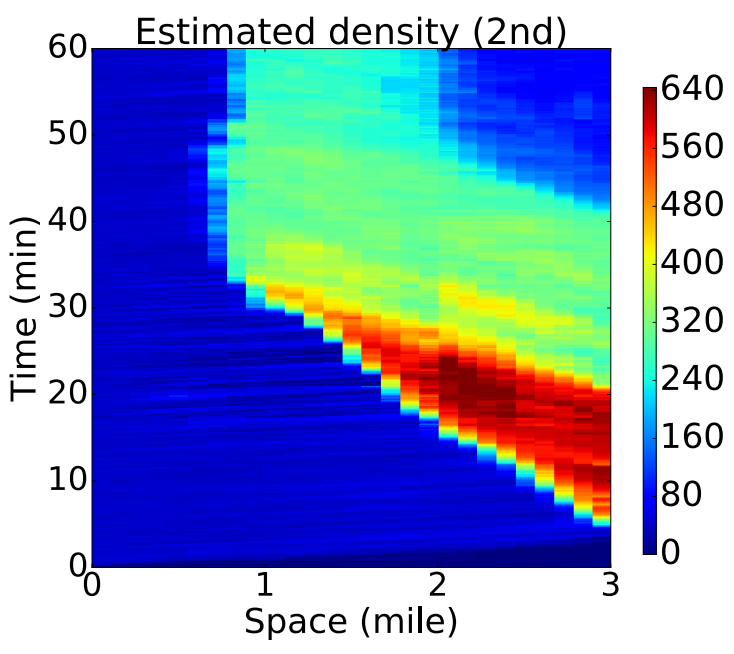

(b)

Figure 2: Estimation results with the fraction of $\mathrm{AVs}$ in the inflow sampled from $\mathcal{U}(0,0)$. (a) Density estimate by the first order model, (b) Density estimate by the second order model.

computation time required (micro simulation, data extraction, and estimation) is about 20 hours for all steps of all presented runs. In addition, the total amount of data generated supporting the presented results is approximately $100 \mathrm{~GB}$. The computational cost of the experiments motivates the exploration of the performance on a simplified geometry, although the algorithms are suitable for more complicated network traffic estimation applications.

\subsection{Results}

The first order model only estimates the total traffic density while the second order model also estimates the fraction of AVs $w$, and is expected to provide more accurate traffic density estimation. Figures 2 and 3 compare the difference between the first and second order models, in two extreme simulation scenarios, where the fractions of AVs in the inflow are sampled from $\mathcal{U}(0,0)$ and $\mathcal{U}(0,1.0)$. The true total densities for two simulation scenarios are plotted in Figure 4.

Figure 2 shows the estimation results for the first order model (Figure 2(a)) and the second order model (Figure 2(b)) when the fraction of AVs is sampled from $\mathcal{U}(0,0)$ (i.e., is constant). Two models perform similarly and both recover the true total density (Figure 4(a)) accurately. This result is expected by recalling that the second order model reduces to the first order model given constant fraction of AVs.

In comparison, Figure 3 shows the estimated total density when the fraction of AVs in the inflow is sampled from $\mathcal{U}(0,1.0)$. The second order model (Figure $3(\mathrm{~b})$ ) captures the extent of the congestion (particularly the expansion wave area) more accurately than the first order model (Figure 3(a)). The better performance of the second order model can be attributed by its capability to estimate the fraction of AVs, as shown in Figure 5. By also 


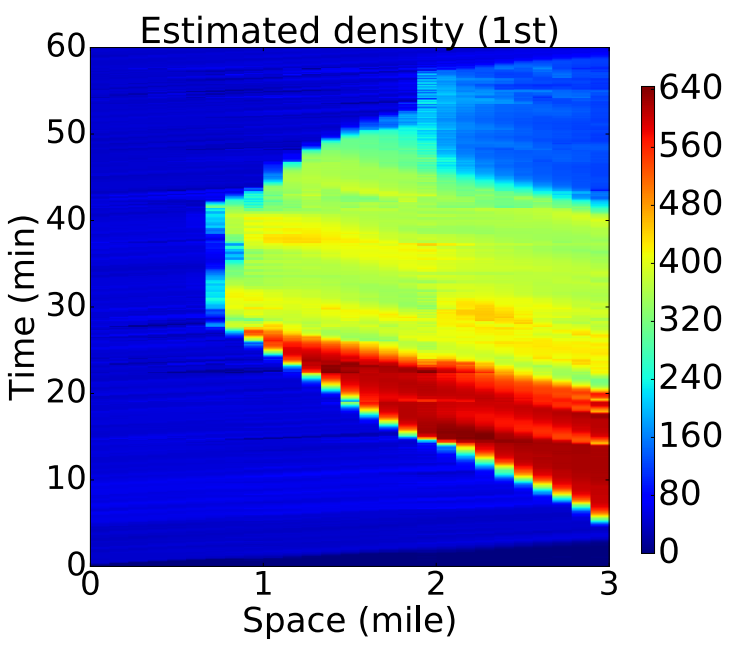

(a)

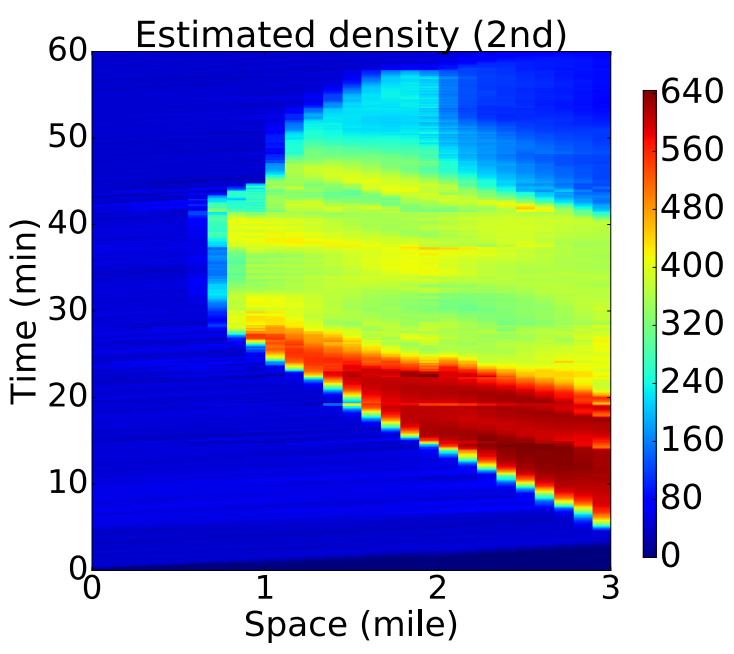

(b)

Figure 3: Estimation results with the fraction of AVs in the inflow sampled from $\mathcal{U}(0,1.0)$. (a) State estimate by the first order model, (b) State estimate by the second order model.

examining the true fraction of AVs shown in Figure 4, Figure 5 shows the second order model can more accurately adjust the fundamental diagram of each cell over the entire time horizon. It should be noted that the erroneous estimate of the fraction of AVs at the initial time (first three minutes) is the warm-up period of the micro simulator and the initialization period of the filtering algorithm, and is neglected in the computation of the error metric for both the first and second order models.

In the next set of experiments, ten replications are simulated for the scenarios with the fraction of $\mathrm{AVs}$ in the inflow sampled from distributions: $\mathcal{U}(0,0), \mathcal{U}(0,0.25), \mathcal{U}(0,0.5)$, $\mathcal{U}(0,0.75), \mathcal{U}(0,1.0)$. More specifically, the $\mathcal{U}(0,0)$ distribution produces inflows with $0 \%$ $\mathrm{AVs}$ and no variability, while the $\mathcal{U}(0,1.0)$ distribution produces inflow with a $50 \%$ mean penetration rate of $\mathrm{AVs}$ and a standard deviation of $29 \%$.

Figure 6 shows the overall mean estimation error as a function of the variability of the penetration rate of $\mathrm{AVs}$ for the first order model estimator (red dash line) and the second order model (blue solid line). Figure 6 illustrates that the second order model estimator generally outperforms the first order model estimator. This is expected since the average model parameter values in the first order model approximate the true model parameters less accurately when the variability of the penetration rates of AVs is large. In comparison, the parameters of the second order model vary depending on the composition of the traffic $w$, resulting in more accurate tracking of the propagation of the congestion.

Next, the normalized improvement of the estimation performance of the second order model over the first order model is also explored. Precisely, in each replication, the normalized improvement of performance is computed as the difference of estimation error between the second order model and the first order model, divided by the estimation error of the first 


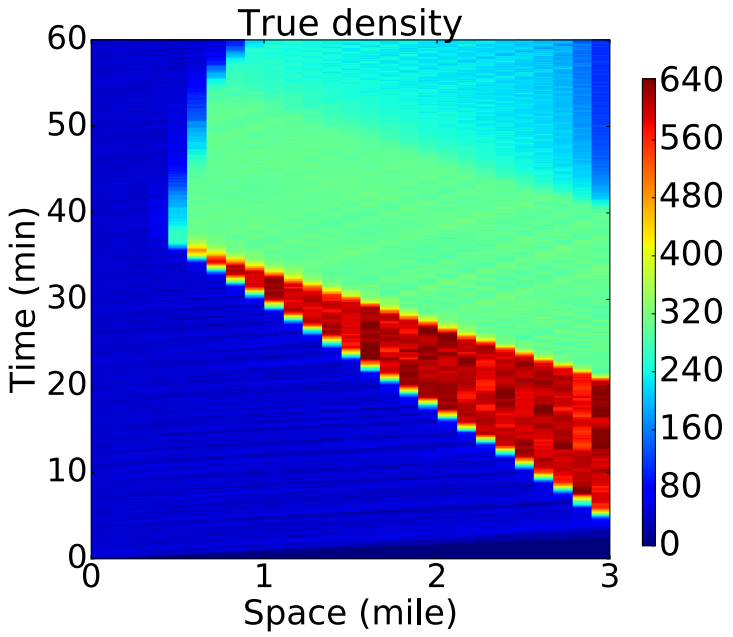

(a)

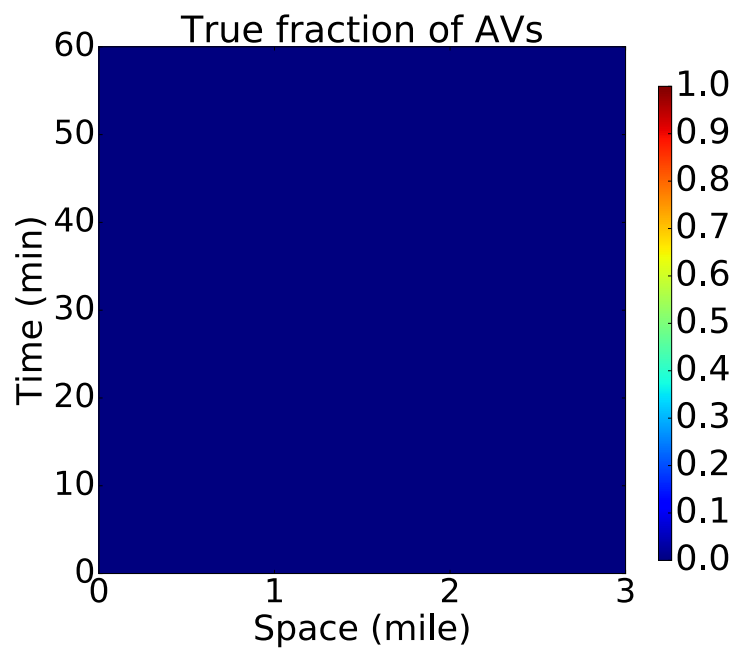

(c)

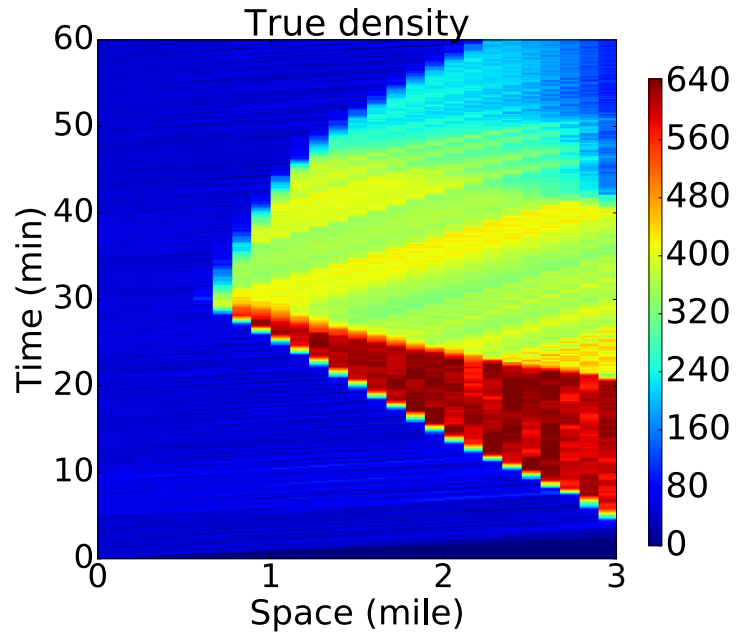

(b)

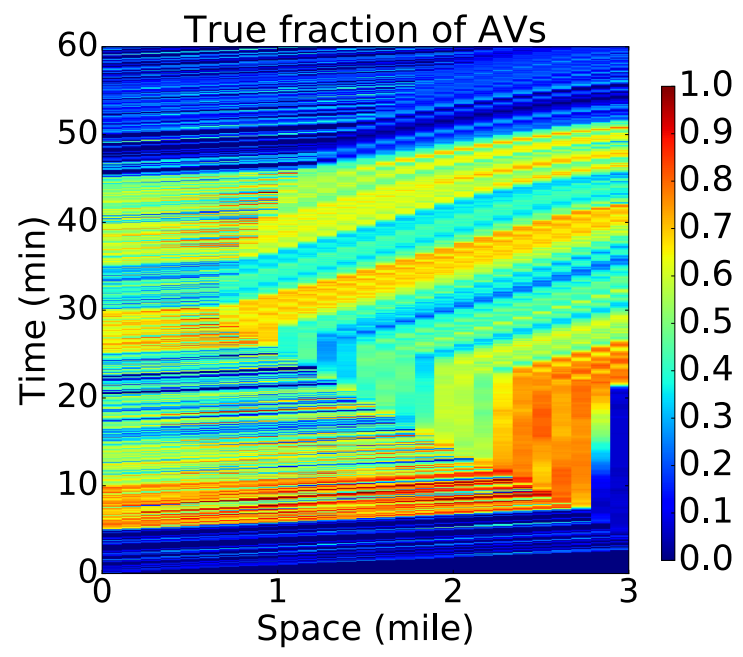

(d)

Figure 4: True total density in two simulation scenarios: (a) when the fraction of AVs in the inflow sampled from $\mathcal{U}(0,0)$, (b) when the fraction of $\mathrm{AVs}$ in the inflow sampled from $\mathcal{U}(0,1.0)$, (c) the true fraction of $\mathrm{AVs}$ in the scenario $\mathcal{U}(0,0),(\mathrm{d})$ the true fraction of $\mathrm{AVs}$ in the scenario $\mathcal{U}(0,1.0)$. 


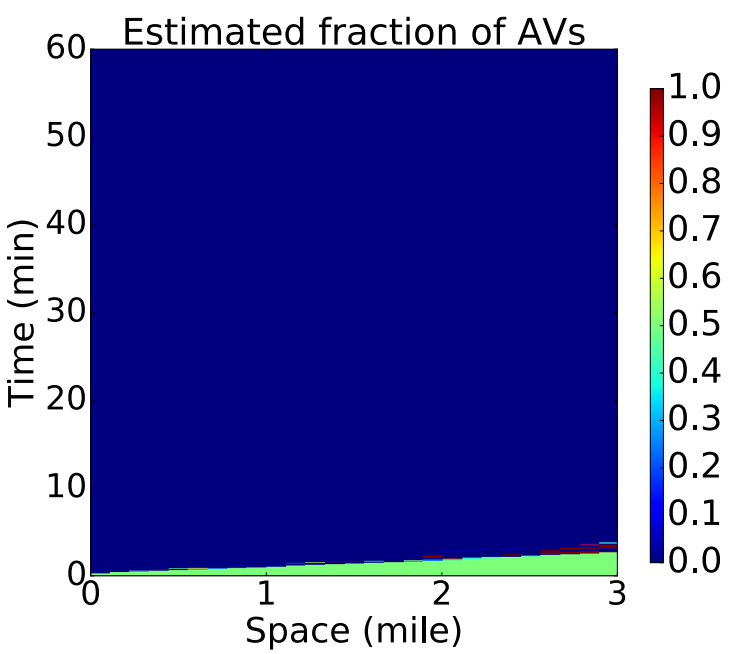

(a)

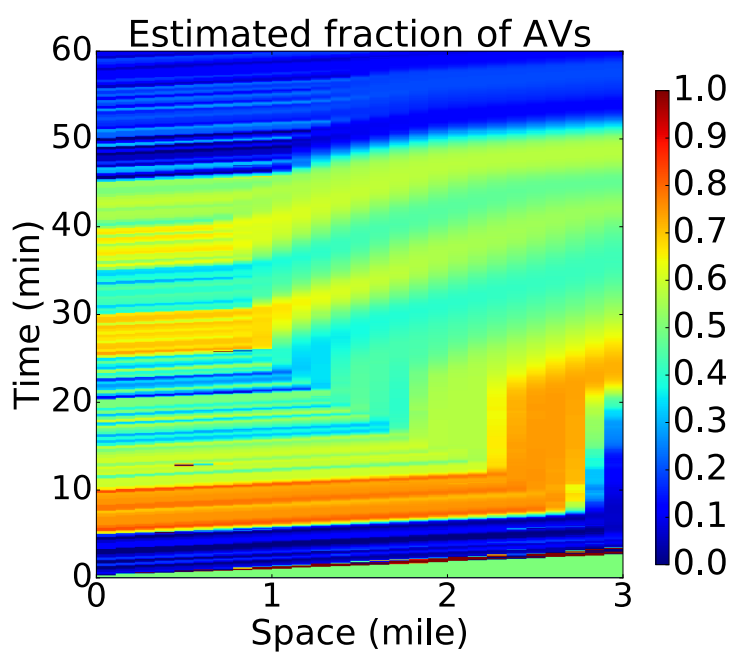

(b)

Figure 5: Estimated fraction of AVs by the second order model estimator: (a) when the fraction of AVs in the inflow is sampled from $\mathcal{U}(0,0)$; (b) when the fraction of AVs in the inflow is sampled from $\mathcal{U}(0,1.0)$. Each estimator is initialized with $w=0.5$, which takes less than 5 minutes to clear the computational domain.

order model. Then, the mean percent improvement is averaged across the ten replications for each scenario, and plotted in Figure 7. It is shown that the second order model estimator outperforms the first order model estimator by up to $17 \%$ for the scenario corresponding to $\mathcal{U}(0,0.75)$.

Finally, a sensitivity analysis is conducted to evaluate the robustness of two estimators. The fundamental diagram parameters $v_{\max }, \rho_{m}$ are perturbed one at a time by $\pm 5 \%$. The critical densities for the first order and second order fundamental diagram are perturbed by $\pm 5 \%,+10 \%$, and $+20 \%$, as initial experiments indicated increasing critical densities resulted in lower estimation error for both models.

The procedure of the sensitivity analysis is as follows: $i$ ) perturb one parameter while keeping other parameters unchanged; ii) run the first order model and second order model using the perturbed set of parameters for ten simulation replications, each with ten particle filter runs; iii) compute the average estimation error of the perturbed set of parameters.

Because of the heavy computational load, the sensitivity analysis was performed for a single penetration rate scenario, $\mathcal{U}(0,0.75)$. The estimation error is plotted in Figure 8 . The x-axis labels denote the perturbed parameter, e.g., $v_{m}^{+5}$ denotes the set of fundamental diagram parameters where $v_{m}$ is perturbed by $+5 \%$. Baseline represents the unperturbed set of parameters.

The performance improvement of the second order model over the first order model for all perturbed set of parameters is shown in Figure 9. As confirmed in the sensitivity analysis, the perturbation of the fundamental diagram parameters indeed varies the performance of the first and second order model estimators. However, regardless of the variation of the 


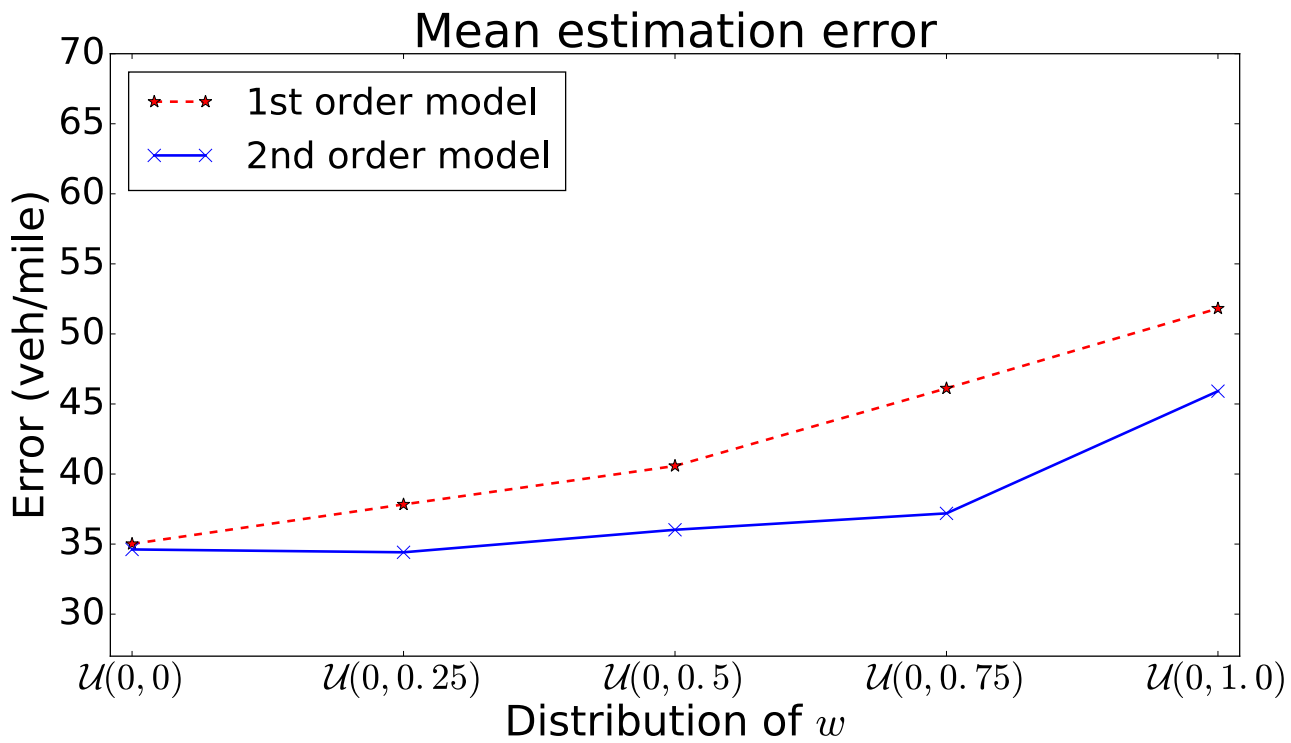

Figure 6: Mean estimation errors for the first order model and second order model in simulation scenarios with different variability of the fraction of AVs.

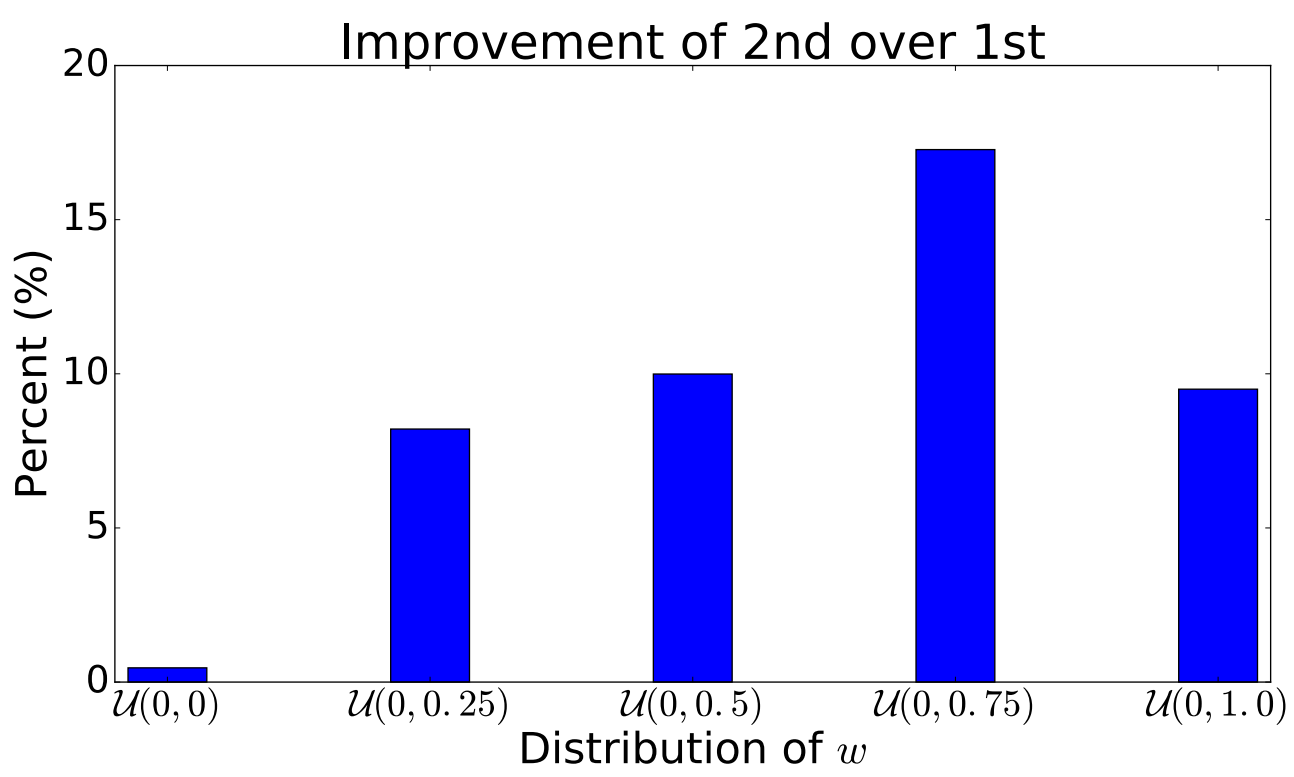

Figure 7: Mean normalized improvement of the performance of the second order model estimator over the first order model estimator at different variability of the fraction of AVs. 


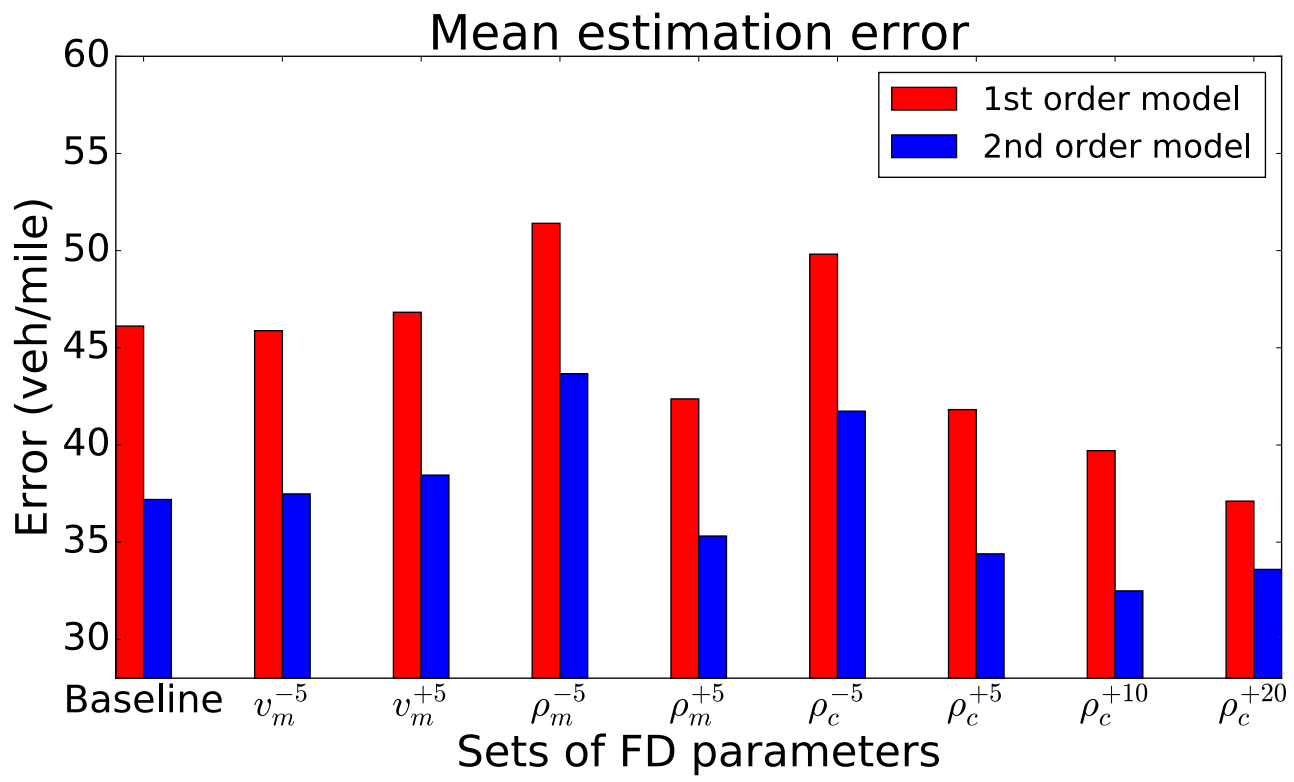

Figure 8: Average estimation error with perturbed set of fundamental diagram parameters.

estimation error, the second order model consistently outperforms the first order model by $9 \%$ to $18 \%$.

\section{Conclusion}

This work explored a connection between the second order traffic flow models and two-class traffic to model flows composed of human piloted and automated vehicles. Based on this connection, a second order model based traffic estimator was compared to a scalar modeling abstraction. For each model, a fully nonlinear particle filter is used to estimate the mixed traffic state generated in a micro simulation environment.

A variety of mixed human and automated traffic scenarios were simulated to evaluate the performance of the estimators. The evaluation results showed the second order model consistently outperformed the first order model in terms of the traffic density accuracy when the variability of the penetration rate increases. At low penetration rate variability, the first order model-based estimator offers similar performance. This empirical finding is consistent with the fact that the second order model reduces to a first order model when the fraction of $\mathrm{AVs}$ is constant.

There are several interesting areas for further exploration. First, the present article assumes that the AVs do not overtake the human piloted traffic, but instead follow the lead vehicle with a smaller average spacing. An open question how human drivers will respond to AVs driving with smaller spacings compared to human piloted traffic. Moreover, the assumption of overtaking may be relaxed if the AVs are extremely aggressive (in which case, 
they become an overtaking class), or passive (in which case they will be passed). Several multi-class traffic flow models which permit overtaking may be appropriate depending on how the AVs are designed to behave in mixed traffic streams.

A second interesting area of further study is the problem of jointly estimating the model parameters and the traffic state online, as was done in [22]. The general difficulty of the online joint parameter and state estimation problem stems from the potential non-observability of the parameters given the observation/measurement equation in the system. If the parameters are observable, it is not necessary to perform the model calibration step offline as was done in the present work.

\section{Acknowledgment}

This material is based upon work supported by the National Science Foundation under Grant No. CNS-1446702.

\section{Appendix}

\section{Cell transmission model}

For numerical implementation in the estimator, (2) is discretized using the standard Godunov scheme [68], (equivalently Cell Transmission Model (CTM) [38, 39, 69]). Specifically, the time and space domains are discretized by introducing a discrete time step $\Delta T$, indexed 
by $n \in\left\{0, \cdots, n_{\max }\right\}$ and a discrete space step $\Delta \chi$, indexed by $i \in\left\{0, \cdots, i_{\max }\right\}$. The discretized system is given by:

$$
\rho_{i}^{n+1}=\rho_{i}^{n}+\frac{\Delta T}{\Delta \chi}\left(G\left(\rho_{i-1}^{n}, \rho_{i}^{n}\right)-G\left(\rho_{i}^{n}, \rho_{i+1}^{n}\right)\right) .
$$

In (18), $\rho_{i}^{n}$ denotes the total traffic density at time step $n$ and in cell $i$. According to equation (18), the traffic density at a cell in the next time step is determined by the traffic density at the cell in the current time step, plus the traffic flow $G\left(\rho_{i-1}^{n}, \rho_{i}^{n}\right)$ that enters from the upstream cell, and minus the traffic flow $G\left(\rho_{i}^{n}, \rho_{i+1}^{n}\right)$ that exits to the downstream cell. The flow (flux) that crosses the cell boundaries is represented by the numerical flux function $G$, which is calculated as the minimum of the traffic flow that can be sent from the upstream cell indicated by $S$, and the traffic flow that can be received by the downstream cell indicated by $R$ :

$$
G\left(\rho_{i}^{n}, \rho_{i+1}^{n}\right)=\min \left\{S\left(\rho_{i}^{n}\right), R\left(\rho_{i+1}^{n}\right)\right\} .
$$

The sending function $S$ and receiving function $R$ are given by:

$$
S\left(\rho_{i}^{n}\right)= \begin{cases}q\left(\rho_{i}^{n}\right) & \text { if } \rho_{i}^{n}<\rho_{c} \\ q\left(\rho_{c}\right) & \text { if } \rho_{i}^{n} \geq \rho_{c}\end{cases}
$$

and

$$
R\left(\rho_{i}^{n}\right)= \begin{cases}q\left(\rho_{c}\right) & \text { if } \rho_{i}^{n}<\rho_{c} \\ q\left(\rho_{i}^{n}\right) & \text { if } \rho_{i}^{n} \geq \rho_{c}\end{cases}
$$

where the flow $q(\rho)=\rho \times v(\rho)$ is the fundamental diagram. To ensure numerical stability, the time and space steps are coupled through the CFL condition [70]: $v_{\max } \frac{\Delta T}{\Delta \chi} \leq 1$.

\section{Second order cell transmission model}

Many approaches are available to discretize second order and multi-class traffic flow models, see for example [71-74]. In this work we follow a standard Godunov approach [71], which requires slightly more care than when it is applied to a first order model. Specifically, a Riemann solver to the PDE (8) is required so the Gudonov fluxs at the cell boundaries can be computed correctly, resulting in a scheme which approximates the correct weak solution. The solution to the Riemann problem is more complicated than the Riemann problem for the LWR model due to the existence of an intermediate state in the solver [57]. In [75], a Riemann solver for the GOSM is constructed through the supply demand framework, which is consistent with the original solver derived by analyzing elementary waves [43, 44]). This results in a second order cell transmission model (2CTM) [71] analogous to the first order CTM. Here, we briefly summarize the scheme as applied to (7) (equivalently (8)). 
where $w_{i}^{n}=y_{i}^{n} / \rho_{i}^{n}=0$.

The first equation provides the evolution of the total density and is identical to the CTM (18) with the exception that the flux function $G_{\rho}$ depends both on the upstream and downstream densities, and additionally the composition of the traffic as indicated by the penetration rate of $\mathrm{AVs} w$ in the upstream and downstream cells. The second equation describes the evolution of the automated vehicle traffic stream $y_{i}^{n}=\rho_{i}^{n} w_{i}^{n}$, and differs from the first system by a flux function $G_{y}$ describing the flow of AVs into and out of the cell over the time step.

An important property of the flow model (7) relates the numerical fluxes $G_{\rho}$ and $G_{y}$. Specifically, since the composition of the flow is advected with the total flow, the flow functions are related by:

$$
G_{y}\left(\rho_{i-1}^{n}, \rho_{i}^{n}, w_{i-1}^{n}, w_{i}^{n}\right)=w_{i-1}^{n} G_{\rho}\left(\rho_{i-1}^{n}, \rho_{i}^{n}, w_{i-1}^{n}, w_{i}^{n}\right) .
$$

This allows the following simplification of the evolution equations (22) that depend only on the flux function $G_{\rho}$ :

$$
\begin{aligned}
\rho_{i}^{n+1}=\rho_{i}^{n} & +\frac{\Delta T}{\Delta \chi} G_{\rho}\left(\rho_{i-1}^{n}, \rho_{i}^{n}, w_{i-1}^{n}, w_{i}^{n}\right) \\
& -\frac{\Delta T}{\Delta \chi} G_{\rho}\left(\rho_{i}^{n}, \rho_{i+1}^{n}, w_{i}^{n}, w_{i+1}^{n}\right), \\
y_{i}^{n+1}=y_{i}^{n} & +\frac{\Delta T}{\Delta \chi} w_{i-1}^{n} G_{\rho}\left(\rho_{i-1}^{n}, \rho_{i}^{n}, w_{i-1}^{n}, w_{i}^{n}\right) \\
& -\frac{\Delta T}{\Delta \chi} w_{i}^{n} G_{\rho}\left(\rho_{i}^{n}, \rho_{i+1}^{n}, w_{i}^{n}, w_{i+1}^{n}\right) .
\end{aligned}
$$

Recall the flow function for the second order model is given as $q(\rho, w)=\rho \times v(\rho, w)$. Unlike the first order flow model, the property variable $w$ is an input to the velocity function 
and the flow function. As a result, the numerical flux is still specified as the minimum of the flow that can be sent and received by the adjacent cells:

$$
G_{\rho}\left(\rho_{i}^{n}, \rho_{i+1}^{n}, w_{i}^{n}, w_{i+1}^{n}\right)=\min \left\{S\left(\rho_{i}^{n}, w_{i}^{n}\right), R\left(\rho_{i+1}^{n}, w_{i}^{n}, w_{i+1}^{n}\right)\right\},
$$

but the sending and receiving functions are more complicated.

The sending function is structurally similar to that in the first order model, and depends on the upstream total density $\rho_{i}^{n}$ and the fraction of AVs $w_{i}^{n}$, which are both inputs to the fundamental diagram and consequently the sending function. Specifically, they are both needed to determine the maximum flow in the sending function, even when a collapsed velocity function is used (i.e., velocity independent of $w$ in light traffic). The receiving function not only depends on the downstream total density $\rho_{i+1}^{n}$, but it also depends on the downstream flow composition $w_{i+1}^{n}$ and the upstream flow composition $w_{i}^{n}$, as will be explained in more detail subsequently. The general form of the sending and receiving functions are:

$$
\begin{gathered}
S\left(\rho_{i}^{n}, w_{i}^{n}\right)= \begin{cases}\rho_{i}^{n} v\left(\rho_{i}^{n}, w_{i}^{n}\right) & \text { if } \rho_{i}^{n}<\rho_{c}\left(w_{i}^{n}\right) \\
\rho_{c}\left(w_{i}^{n}\right) v\left(\rho_{c}\left(w_{i}^{n}\right), w_{i}^{n}\right) & \text { if } \rho_{i}^{n}<\rho_{c}\left(w_{i}^{n}\right),\end{cases} \\
R\left(\rho_{i+1}^{n}, w_{i}^{n}, w_{i+1}^{n}\right)= \begin{cases}\rho_{c}\left(w_{i}^{n}\right) v\left(\rho_{c}\left(w_{i}^{n}\right), w_{i}^{n}\right) & \text { if } \rho_{M}<\rho_{c}\left(w_{i}^{n}\right) \\
\rho_{M}\left(w_{i}^{n}, w_{i+1}^{n}\right) v\left(\rho_{M}, w_{i}^{n}\right) & \text { if } \rho \geq \rho_{c}\left(w_{i}^{n}\right),\end{cases}
\end{gathered}
$$

where $\rho_{M}$ is an intermediate total traffic density. For the collapsed velocity function (11) where the free flow velocity does not depend on the traffic composition, the intermediate density can be calculated as:

$$
\rho_{M}=\underset{\rho}{\operatorname{argmin}} v\left(\rho_{i+1}, w_{i+1}\right)-v\left(\rho, w_{i}\right),
$$

where the time index $n$ is dropped for brevity.

Roughly, the intermediate density $\rho_{M}$ can be interpreted as the average (total) density of traffic when traveling at the speed of traffic $v\left(\rho_{i+1}^{n}, w_{i+1}^{n}\right)$ defined in the downstream cell, with the same penetration rate of $\mathrm{AVs} w_{i}^{n}$ as in the upstream cell. This interpretation is a result of the fact that the composition of traffic $w$ is advected with the flow and therefore is defined by the upstream cell, and the speed of traffic is defined from the downstream cell because overtaking does not occur. It is logical that the amount of vehicles that can be received downstream should depend both on the downstream traffic composition as well as the intermediate density, which captures the composition of the entering vehicles.

A final note is required to address the special case when the total density $\rho_{i}^{n}=0$ in a cell. Because the density of automated vehicles $y_{i}^{n}$ is also 0 , the variable $w_{i}^{n}=y_{i}^{n} / \rho_{i}^{n}$ is undefined. In order for the correct cell boundary flow to be computed (i.e., the one consistent with the solution to the Riemann problem for $(7)$ ), the value of $w$ should be set as 1 . This allows the sending flow out of the $i^{\text {th }}$ cell (on the downstream boundary) to be correctly computed as zero in (24). It also allows the receiving function (25) into the $i^{\text {th }}$ cell (on the upstream boundary) to be maximal, and the maximum flow which can be sent from the $i-1^{\text {th }}$ cell will determine the inflow. 


\section{Particle filter}

A summary of the particle filter is presented in Algorithm $1[61,62]$. Let $M$ denote the number of particles used in the filter, which are indexed by $l$. Let $\zeta_{l}$ denote the weight of particle $l$. In the initial time step, a number of particles are generated based on the initial knowledge of the highway traffic state. Next, the particles are evolved forward in time to compute the predicted traffic states for the next time step. The predicted traffic states $x_{l}^{n}$ are known as the prior distribution. Then, after the measurements $z^{n}$ are received, the measurements from the field (i.e., density measurements) are used to determine the likelihood of each predicted traffic state. The particles are weighted according to the computed likelihood at the current time step and their previous weights. The weights of the particles are normalized so that the sum of the particle weights equals to one. Finally, resampling is performed so that the samples with high weights are kept and duplicated and the samples with low weights are removed from the sample set. The output of a particle filter is the posterior distribution of the system state $x$ at each time step $n$, which is the estimated traffic state at each time step.

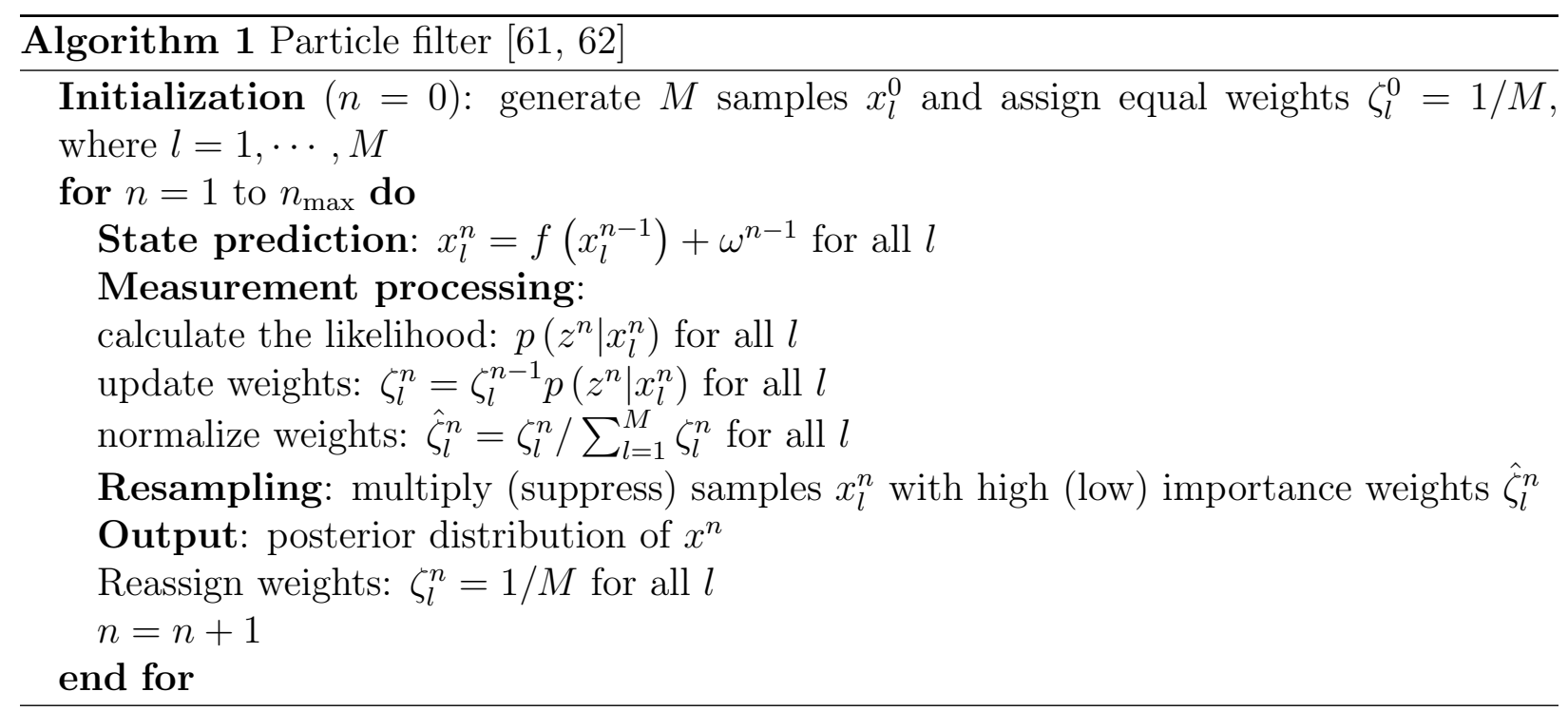


1] R. E. Fenton and R. J. Mayhan. Automated highway studies at the Ohio State University-an overview. IEEE transactions on Vehicular Technology, 40(1):100-113, 1991.

[2] J. G. Bender. An overview of systems studies of automated highway systems. IEEE Transactions on Vehicular Technology, 40(1):82-99, 1991.

[3] S. E. Shladover, C. A. Desoer, J. K. Hedrick, M. Tomizuka, J. Walrand, W.-B. Zhang, D. H. McMahon, H. Peng, S. Sheikholeslam, and N. McKeown. Automated vehicle control developments in the PATH program. IEEE Transactions on vehicular technology, 40(1):114-130, 1991.

[4] S. E. Shladover. Review of the state of development of advanced vehicle control systems (AVCS). Vehicle System Dynamics, 24(6-7):551-595, 1995.

[5] P. Ioannou. Automated highway systems. Springer Science \& Business Media, 2013.

[6] B. Van Arem, C. J. G. Van Driel, and R. Visser. The impact of cooperative adaptive cruise control on traffic-flow characteristics. IEEE Transactions on Intelligent Transportation Systems, 7(4):429-436, 2006.

[7] M. Buehler, K. Iagnemma, and S. Singh. The DARPA urban challenge: autonomous vehicles in city traffic, volume 56. springer, 2009.

[8] A. Bose and P. Ioannou. Analysis of traffic flow with mixed manual and semiautomated vehicles. IEEE Transactions on Intelligent Transportation Systems, 4(4):173-188, 2003.

[9] K. Li and P. Ioannou. Modeling of traffic flow of automated vehicles. IEEE Transactions on Intelligent Transportation Systems, 5(2):99-113, 2004.

[10] S. Shladover, D. Su, and X.-Y. Lu. Impacts of cooperative adaptive cruise control on freeway traffic flow. Transportation Research Record: Journal of the Transportation Research Board, 2324:63-70, 2012.

[11] C. Diakaki, M. Papageorgiou, I. Papamichail, and I. Nikolos. Overview and analysis of vehicle automation and communication systems from a motorway traffic management perspective. Transportation Research Part A: Policy and Practice, 75:147-165, 2015.

[12] D. Ngoduy. Instability of cooperative adaptive cruise control traffic flow: a macroscopic approach. Communications in Nonlinear Science and Numerical Simulation, 18(10):2838-2851, 2013.

[13] D. Ngoduy. Application of gas-kinetic theory to modelling mixed traffic of manual and ACC vehicles. Transportmetrica, 8(1):43-60, 2012.

[14] M. W. Levin and S. D. Boyles. A multiclass cell transmission model for shared human and autonomous vehicle roads. Transportation Research Part C: Emerging Technologies, 62:103 - 116, 2016.

[15] A. I. Delis, I. K. Nikolos, and M. Papageorgiou. Macroscopic traffic flow modeling with adaptive cruise control: Development and numerical solution. Computers \& Mathematics with Applications, 70(8):1921-1947, 2015.

[16] A. Talebpour and H. S. Mahmassani. Influence of autonomous and connected vehicles on stability of traffic flow. In Proceedings of the Transportation Research Board 94th Annual Meeting, number 15-5971, 2015.

[17] L. C. Davis. Effect of adaptive cruise control systems on traffic flow. Physical Review E, 69(6):066110, 2004.

[18] D. C. Gazis and C. H. Knapp. On-line estimation of traffic densities from time-series of flow and speed data. Transportation Science, 5(3):283-301, 1971.

[19] M. Szeto and D. Gazis. Application of Kalman filtering to the surveillance and control of traffic systems. Transportation Science, 6(4):419-439, 1972

[20] M. Cremer and M. Papageorgiou. Parameter identification for a traffic flow model. Automatica, $17(6): 837-843,1981$.

[21] M. Papageorgiou. Applications of Automatic Control Concepts to Traffic Flow Modeling and Control. Springer-Verlag New York, 1983.

[22] Y. Wang and M. Papageorgiou. Real-time freeway traffic state estimation based on extended Kalman filter: a general approach. Transportation Research Part B: Methodological, 39(2):141-167, 2005.

[23] Y. Wang, M. Papageorgiou, A. Messmer, P. Coppola, A. Tzimitsi, and A. Nuzzolo. An adaptive freeway traffic state estimator. Automatica, 45(1):10-24, 2009.

[24] S. Blandin, A. Couque, A. Bayen, and D. Work. On sequential data assimilation for scalar macroscopic 
[25] D. Work, S. Blandin, O. P. Tossavainen, B. Piccoli, and A. M. Bayen. A traffic model for velocity data assimilation. Applied Mathematics Research eXpress, 2010(1):1-35, 2010.

[26] X. Sun, L. Muñoz, and R. Horowitz. Mixture Kalman filter based highway congestion mode and vehicle density estimator and its application. In Proceedings of the American Control Conference, volume 3, pages 2098-2103, 2004.

[27] Y. Sun and D. Work. A distributed local kalman consensus filter for traffic estimation. In Proceedings of the IEEE Conference on Decision and Control, pages 6484-6491, 2014.

[28] L. Mihaylova, R. Boel, and A. Hegyi. An unscented Kalman filter for freeway traffic estimation. volume 39, pages 31-36. Elsevier, 2006.

[29] S. E. Jabari and H. Liu. A stochastic model of traffic flow: Gaussian approximation and estimation. Transportation Research Part B: Methodological, 47:15-41, 2013.

[30] L. Mihaylova and R. Boel. A particle filter for freeway traffic estimation. In Proceedings of the IEEE Conference on Decision and Control, pages 2106-2111, 2004.

[31] L. Mihaylova, R. Boel, and A. Hegyi. Freeway traffic estimation within particle filtering framework. Automatica, 43(2):290-300, 2007.

[32] J. Sau, N. El Faouzi, A. B. Assa, and O. De Mouzon. Particle filter-based real-time estimation and prediction of traffic conditions. Applied Stochastic Models and Data Analysis, 12, 2007.

[33] N. Polson and V. Sokolov. Bayesian analysis of traffic flow on interstate I-55: The LWR model. arXiv preprint arXiv:1409.6034, 2014.

[34] H. Chen, H. A. Rakha, and S. Sadek. Real-time freeway traffic state prediction: A particle filter approach. Proceedings of the IEEE conference Intelligent Transportation Systems, pages 626-631, 2011.

[35] R. Wang, D. B. Work, and R. Sowers. Multiple model particle filter for traffic estimation and incident detection. IEEE Transactions on Intelligent Transportation Systems, PP(99):1-10, 2016.

[36] M. J. Lighthill and G. B. Whitham. On kinematic waves II. a theory of traffic flow on long crowded roads. In Proceedings of the Royal Society of London A: Mathematical, Physical and Engineering Sciences, volume 229, pages 317-345, 1955.

[37] P. I. Richards. Shock waves on the highway. Operations Research, 4(1):42-51, 1956.

[38] C. F. Daganzo. The cell transmission model: A dynamic representation of highway traffic consistent with the hydrodynamic theory. Transportation Research Part B: Methodological, 28(4):269-287, 1994.

[39] C. F. Daganzo. The cell transmission model, part II: network traffic. Transportation Research Part B: Methodological, 29(2):79-93, 1995.

[40] N. Bekiaris-Liberis, C. Roncoli, and M. Papageorgiou. Highway traffic state estimation with mixed connected and conventional vehicles using speed measurements. In Proceedings of the IEEE International Conference on Intelligent Transportation Systems, pages 2806-2811, 2015.

[41] N. Bekiaris-Liberis, C. Roncoli, and M. Papageorgiou. Highway traffic state estimation with mixed connected and conventional vehicles. IEEE Transactions on Intelligent Transportation Systems, 99:1$14,2016$.

[42] C. Roncoli, N. Bekiaris-Liberis, and M. Papageorgiou. Highway traffic state estimation using speed measurements: Case studies on NGSIM data and Highway A20 in the Netherlands. In in Proceedings of the Transportation Research Board 95th Annual Meeting, number 16-2071, 2016.

[43] A. Aw and M. Rascle. Resurrection of second order models of traffic flow. SIAM Journal on Applied Mathematics, 60:916-944, 2000.

[44] H. M. Zhang. A non-equilibrium traffic model devoid of gas-like behavior. Transportation Research Part B: Methodological, 36:275-290, 2002.

[45] Y. Yuan, J. W. C. Van Lint, R. E. Wilson, F. Van Wageningen-Kessels, and S. P. Hoogendoorn. Realtime Lagrangian traffic state estimator for freeways. IEEE Transactions on Intelligent Transportation Systems, 13(1):59-70, March 2012.

[46] T. Seo, T. Kusakabe, and Y. Asakura. Estimation of flow and density using probe vehicles with spacing measurement equipment. Transportation Research Part C: Emerging Technologies, 53:134-150, 2015.

[47] S. Fan and B. Seibold. A comparison of data-fitted first order traffic models and their second order 
[48] S. Fan and D. Work. A heterogeneous multiclass traffic flow model with creeping. SIAM Journal on Applied Mathematics, 75(2):813-835, 2015.

[49] J. Van Lint, S. Hoogendoorn, and M. Schreuder. Fastlane: New multiclass first-order traffic flow model. Transportation Research Record: Journal of the Transportation Research Board, (2088):177-187, 2008.

[50] D. Ngoduy and R. Liu. Multiclass first-order simulation model to explain non-linear traffic phenomena. Physica A: Statistical Mechanics and its Applications, 385(2):667-682, 2007.

[51] S. Benzoni-Gavage and R. M. Colombo. An $n$-populations model for traffic flow. European Journal of Applied Mathematics, 14(05):587-612, 2003.

[52] D. Helbing, A. Hennecke, V. Shvetsov, and M. Treiber. MASTER: macroscopic traffic simulation based on a gas-kinetic, non-local traffic model. Transportation Research Part B: Methodological, 35(2):183211, 2001.

[53] M. Guériau, R. Billot, N.-E. El Faouzi, J. Monteil, F. Armetta, and S. Hassas. How to assess the benefits of connected vehicles? a simulation framework for the design of cooperative traffic management strategies. Transportation Research Part C: Emerging Technologies, 67:266 - 279, 2016.

[54] S. Fan, M. Herty, and B. Seibold. Comparative model accuracy of a data-fitted generalized Aw-RascleZhang model. Networks and Heterogeneous Media, Vol. 9, No. 2:239-268, 2014.

[55] S. Smulders. Control of freeway traffic flow by variable speed signs. Transportation Research Part B: Methodological, 24(2):111-132, 1990.

[56] J. P. Lebacque, S. Mammar, and H. Haj-Salem. Generic second order traffic flow modelling. In Transportation and Traffic Theory, pages 755-776, 2007.

[57] P. Zhang, S. C. Wong, and S. Q. Dai. A conserved higher-order anisotropic traffic flow model: description of equilibrium and non-equilibrium flows. Transportation Research Part B: Methodological, 43(5):562-574, 2009.

[58] S. Blandin, D. Work, P. Goatin, B. Piccoli, and A. Bayen. A general phase transition model for vehicular traffic. SIAM Journal on Applied Mathematics, 71(1):107-127, 2011.

[59] H. Zhang and W. Jin. Kinematic wave traffic flow model for mixed traffic. Transportation Research Record: Journal of the Transportation Research Board, (1802):197-204, 2002.

[60] S. Fan. Data-Fitted Generic Second Order Macroscopic Traffic Flow Models. PhD thesis, Temple University, 2013.

[61] B. Ristic, S. Arulampalm, and N. Gordon. Beyond the Kalman filter: Particle filters for tracking applications. Artech House Publishers, 2004.

[62] A. Doucet and A. M. Johansen. A tutorial on particle filtering and smoothing: Fifteen years later. Handbook of nonlinear filtering, 12(656-704):3, 2009.

[63] J. P. Kaipio and E. Somersalo. Statistical and computational inverse problems. Springer, 2005.

[64] I. A. Ntousakis, I. K. Nikolos, and M. Papageorgiou. On microscopic modelling of adaptive cruise control systems. Transportation Research Procedia, 6:111 - 127, 2015.

[65] R. Rajamani and S. E. Shladover. An experimental comparative study of autonomous and co-operative vehicle-follower control systems. Transportation Research Part C: Emerging Technologies, 9(1):15 - 31, 2001.

[66] K. Li and P. Ioannou. Modeling of traffic flow of automated vehicles. IEEE Transactions on Intelligent Transportation Systems, 5(2):99-113, 2004.

[67] L. C. Edie. Discussion of traffic stream measurements and definitions. In Proceedings of the 2nd International Symposium on the Theory of Road Traffic Flow, pages 139-154, 1965.

[68] S. K. Godunov. A difference method for numerical calculation of discontinuous solutions of the equations of hydrodynamics. Matematicheskii Sbornik, 89(3):271-306, 1959.

[69] J. P. Lebacque. Les modeles macroscopiques de trafic. In Annales des Ponts et chaussées, number 67, pages 24-45, 1993.

[70] R. J. LeVeque. Numerical Methods for Conservation Laws. Birkhäuser, 1992.

[71] S. Fan, Y. Sun, B. Piccoli, B. Seibold, and D. Work. A collapsed generalized Aw-Rascle-Zhang model and its model accuracy. in preparation, 2016. 
[72] G. C. K. Wong and S. C. Wong. A multi-class traffic flow model-an extension of LWR model with heterogeneous drivers. Transportation Research Part A: Policy and Practice, 36(9):827-841, 2002.

[73] M. Zhang, C. W. Shu, G. C. K. Wong, and S. C. Wong. A weighted essentially non-oscillatory numerical scheme for a multi-class Lighthill-Whitham-Richards traffic flow model. Journal of Computational Physics, 191(2):639-659, 2003.

[74] F. van Wageningen-Kessels, H. van Lint, S. Hoogendoorn, and K. Vuik. Lagrangian formulation of multiclass kinematic wave model. Transportation Research Record: Journal of the Transportation Research Board, (2188):29-36, 2010.

[75] J. P. Lebacque, H Haj-Salem, and S. Mammar. Second order traffic flow modeling: supply-demand analysis of the inhomogeneous Riemann problem and of boundary conditions. In Proceedings of the 10th Euro Working Group on Transportation (EWGT), pages 108-115, 2005. 